\title{
Overexpression of TMPRSS4 promotes tumor proliferation and aggressiveness in breast cancer
}

\author{
XIAO-MEI LI ${ }^{1,2^{*}}$, WEN-LOU LIU ${ }^{3 *}$, XU CHEN $^{1}$, YA-WEN WANG ${ }^{1}$, DUAN-BO SHI ${ }^{1}$, HUI ZHANG ${ }^{1}$, \\ RAN-RAN MA ${ }^{1}$, HAI-TING LIU ${ }^{1}$, XIANG-YU GUO ${ }^{1}$, FENG HOU $^{4}$, MING LI $^{5}$ and PENG GAO ${ }^{1}$ \\ ${ }^{1}$ Department of Pathology, Qilu Hospital of Shandong University, Jinan, Shandong 250012; ${ }^{2}$ Department of Pathology, \\ Taian Central Hospital, Taian, Shandong 271000; ${ }^{3}$ Department of Oncology, Zhongnan Hospital of Wuhan University, \\ Wuhan, Hubei 430071; ${ }^{4}$ Department of Pathology, Affiliated Hospital of Qingdao University, Qingdao, Shandong 266000; \\ ${ }^{5}$ Department of Pathology, Dezhou Renmin Hospital, Dezhou, Shandong 253000, P.R. China
}

Received June 19, 2016; Accepted February 10, 2017

DOI: 10.3892/ijmm.2017.2893

\begin{abstract}
Transmembrane protease serine 4 (TMPRSS4) is a novel type II transmembrane serine protease that is overexpressed in various types of human cancers and has an important function in cancer progression. However, there is a paucity of data available regarding the biological effects of TMPRSS4 on breast cancer (BC) cells and the underlying mechanisms. In this study, expression of TMPRSS4 in BC tissues was detected by immunohistochemistry. The relationship between TMPRSS4 expression and clinicopathological characteristics as well as prognosis was evaluated. The effects of TMPRSS4 on cell proliferation, migration and invasion were investigated in BC cell lines in vitro. Additionally, RT-qPCR and western blot analysis were used to determine the expressions of epithelial-mesenchymal transition (EMT) biomarkers and TMPRSS4 in BC cell lines. We found that TMPRSS4 was overexpressed in $\mathrm{BC}$ tissues and its expression level was closely correlated with tumor size, histological grade, lymph node metastasis, clinical stage as well as poor survival (all $\mathrm{P}<0.05$ ) and could be recognized as an independent prognostic factor for BC patients. Overexpression of TMPRSS4 promoted the proliferation, migration and invasion of $\mathrm{BC}$ cells in vitro. Moreover, TMPRSS4 knockdown significantly enhanced the expression of E-cadherin and claudin-1 and inhibited the expression of vimentin and Slug, indicating suppression of EMT. Our results suggest that TMPRSS4 plays a crucial role in the progression of BC. Moreover, TMPRSS4 overexpression promoted the proliferation, invasion and migration of $\mathrm{BC}$
\end{abstract}

Correspondence to: Professor Peng Gao, Department of Pathology, Qilu Hospital of Shandong University, 107 West Culture Road, Jinan, Shandong 250012, P.R. China

E-mail: gaopeng@sdu.edu.cn

${ }^{*}$ Contributed equally

Key words: breast cancer, transmembrane protease serine 4, proliferation, invasion, epithelial-mesenchymal transition, serine protease cells by possibly inducing EMT. To conclude, TMPRSS4 may be a potential therapeutic target for cancer treatment.

\section{Introduction}

Breast cancer $(\mathrm{BC})$ is one of the most common cancers and the leading cause of morbidity among all female malignant tumors, despite improvements in screening and adjuvant systemic treatment (1). It has been widely acknowledged that $\mathrm{BC}$ is a highly heterogeneous disease, with different biological behaviors for the same stage of BC among different patients (2). Therefore, personalized therapy based on the different tumor molecular classifications has become the main research field in BC treatment (3). To date, personalized therapy of BC is still guided by various traditional prognostic and predictive parameters, including estrogen receptor (ER) and progesterone receptor (PR) status, and human epidermal growth factor receptor 2 (HER2) amplification (4,5). Therefore, there is an urgent requirement to identify new prognostic and predictive biomarkers that can be used to optimize treatments and predict clinical outcomes among BC patients.

Previous studies have indicated that serine proteases play an important role in cancer invasion and metastasis. Proteases contribute to degradation of the basement membrane and extracellular matrix (ECM) $(6,7)$, which allows tumor cells to invade the surrounding tissue and nearby blood vessels. In addition, proteases may be involved in all stages of the development and progression of cancer, including proliferation, survival, migration, invasion, angiogenesis and metastasis (8). Type II transmembrane serine proteases (TTSPs) are a new subfamily of serine proteases that participate in the regulation of cellular signaling events at the plasma membrane and in the ECM $(9,10)$. Many of the TTSPs have been found to be dysregulated in malignant tumors, implicating their possible roles in tumorigenesis and/or progression (11).

Transmembrane protease serine 4 (TMPRSS4) is a novel TTSP expressed at the cell surface. It is overexpressed in pancreatic, thyroid, colon, lung and gastric cancer tissues and is associated with poor patient prognosis (12), suggesting a possible role for TMPRSS4 in tumor development and 
progression. Recent studies suggest that TMPRSS4 can induce epithelial-mesenchymal transition (EMT), accompanied by increased invasive activity and malignant transformation in human epithelial cancer cells (13-15). Given these findings, TMPRSS4 appears to play important roles in carcinogenesis and may represent a new therapeutic target for cancers. It has also been reported that TMPRSS4 is overexpressed in $\mathrm{BC}$ tissue, but few studies have documented the function of TMPRSS4 and its underlying mechanism in BC cell lines. In the present study, we examined TMPRSS4 expression in $\mathrm{BC}$ tissues and its correlation with clinicopathological parameters and prognosis. Moreover, we detected the function of TMPRSS4 in breast cancer cell lines and key biomarkers in EMT to further investigate the relationship between TMPRSS4 and EMT in BC.

\section{Materials and methods}

Clinical specimens. One hundred and seven formalin-fixed, paraffin-embedded (FFPE) breast cancer (BC) specimens and 52 normal breast tissue samples were obtained from the Department of Pathology, Taian Central Hospital between January 2008 and December 2009. All patients included in the present study received no radiotherapy or chemotherapy prior to surgical resection. Major patient demographic and clinicopathological characteristics were available, including age, menopausal status, tumor size, lymph node (LN) status, histological subtype, tumor grade, clinical stage, and ER, PR and HER2 status. Males were excluded and all patients were females. TNM stage and histological grade were classified according to the 7th edition of American Joint Committee on Cancer (AJCC) TNM system (16) and the 4th edition of WHO histological grade (17). The study protocol was approved by the Institutional Ethics Committee of Taian Central Hospital and informed consent was obtained from all patients. The study was undertaken according to the ethical standards of the World Medical Association Declaration of Helsinki.

Follow-up information was obtained from the medical records or by phone call. All patients had follow-up records for $>5$ years. The follow-up deadline was June 2015. Disease-free survival (DFS) time and overall survival (OS) time were calculated from the date of surgery to the date of first recurrence or death, which were the two assessments used for prognostic analyses.

Immunohistochemistry and evaluation. Immunohistochemical analysis of breast tissues was performed on formalinfixed, paraffin-embedded, $4-\mu$ m-thick tissue sections using the (ABC) avidin-biotin-peroxidase complex method. Briefly, the sections were deparaffinized and dehydrated using a graded series of ethanol solutions. Antigen retrieval was carried out by treatment in a microwave in a $0.01 \mathrm{M}$ citrate buffer ( $\mathrm{pH}$ 6.0). The sections were incubated with the primary rabbit anti-TMPRSS4 antibody (1:200; ab150595, Abcam, Cambridge, UK) overnight at $4^{\circ} \mathrm{C}$ followed by the secondary antibody. The primary antibody was replaced with Tris-buffered saline to act as the negative control. The results were visualized with diaminobenzidine, and all sections were counterstained with hematoxylin and differentiated by hydrochloric acid alcohol.
The immunostaining results were determined independently by two expert pathologists (X.-M.L., X.C.) in a double-blind manner. The staining intensity was classified as follows: 0 , no staining; 1 , weak staining; 2 , moderate staining; 3 , strong staining. The percentage of positive-stained tumor cells was scored as follows: 0 , none; $1,<15 \%$ positive tumor cells; $2,15-50 \%$ positive tumor cells; and $3,>50 \%$ positive tumor cells. The staining index (SI) was calculated as staining intensity score $\mathrm{x}$ proportion of positive tumor cells. SI scores of 0-3 were considered low expression and $>3$ were considered high expression.

Cell culture. All five human breast cancer cell lines, MCF-7, T47D, ADM, MDA-MB-468 and MDA-MB-231 were purchased from Shanghai Cancer Institute and preserved at the Department of Pathology of Shandong University. MCF-7 cells were maintained in Dulbecco's Modified Eagle's Medium (DMEM) with $10 \%$ fetal bovine serum (FBS). T47D and ADM cells were cultured in RPMI-1640 medium containing 10\% FBS. MDA-MB-468 and MDA-MB-231 cells were cultured in L-15 medium containing $10 \%$ FBS. All media and FBS were purchased from Gibco (Los Angeles, CA, USA). Cells were incubated at $37^{\circ} \mathrm{C}$ with $5 \% \mathrm{CO}_{2}$.

Transient transfection. Commercial siRNA targeting TMPRSS4 was purchased from RiboBio Co., Ltd.(Guangzhou, China). Cells were seeded in 6-well culture plates (Nest Biotechnology Co., Ltd., Wuxi, China) at a density of $5 \times 10^{5}$ cells/well and transfected with siRNA and negative controls (NCs) by X-tremeGENE transfection reagent $12 \mathrm{~h}$ later (Roche Applied Science, Indianapolis, IN, USA). Forty-eight hours after transfection, quantitative real-time PCR (RT-qPCR) and western blot analysis were used to examine transfection efficiency. Cell function assays, RNA isolation and total cell protein extraction were performed $48 \mathrm{~h}$ after transfection.

$R N A$ isolation and reverse transcription-quantitative polymerase chain reaction $(R T-q P C R)$. Total RNA was isolated using the TRIzol agent (Invitrogen, Carlsbad, CA, USA) and RNA samples $(1 \mu \mathrm{g})$ were reverse transcripted to cDNA using PrimeScript RT Master Mix (538100; Toyobo, Osaka, Japan). The qPCR was performed using SYBR-Green PCR Master Mix (15153900; Roche, Indianapolis, IN, USA) on the Applied Biosystems 7900HT Real-time PCR system. Glyceraldehyde 3-phosphate dehydrogenase (GAPDH) mRNA was used as an internal control for each sample, and the expression of each sample was normalized to GAPDH mRNA. Primers used were as follows: TMPRSS4 forward, 5'-CCGATGTGTTC AACTGGAAG-3' and reverse, 5'-GAGAAAGTGAGTGG GAACTG-3'; GAPDH forward, 5'-GCACCGTCAAGGCTG AGAAC-3' and reverse, 5'-TGGTGAAGACGCCAGTGGA-3'. After denaturation for $10 \mathrm{~min}$ at $95^{\circ} \mathrm{C}$, the reaction was continued for $40 \mathrm{cycles}$ at $94^{\circ} \mathrm{C}$ for $10 \mathrm{sec}, 60$ or $62^{\circ} \mathrm{C}$ for $20 \mathrm{sec}$ and $72^{\circ} \mathrm{C}$ for $20 \mathrm{sec}$. The relative expression of each miRNA was calculated using the $2^{-\Delta \Delta \mathrm{Ct}}$ method.

Western blot analysis. Total protein was extracted from the transfected cells $48 \mathrm{~h}$ after transfection and protein concentration was determined by the BCA protein assay kit (Blue Skies, Shanghai, China). Primary antibodies including 
TMPRSS4 (1:1,000; ab82176, Abcam), E-cadherin, claudin-1, vimentin, Slug and ZEB1 (1:1,000; mAb 3195, mAb 13255, mAb 5741, mAb 9585, mAb 3396, Cell Signaling Technology, Danvers, MA, USA) from the EMT antibody sampler kit (1:1,000; Cell Signaling Technology) were incubated overnight at $4^{\circ} \mathrm{C}$, washed, and then incubated with horseradish peroxidase-labelled secondary anti-rabbit $\operatorname{IgG}(1: 5,000$; antibody 7074, Cell Signaling Technology) for $30 \mathrm{~min}$. Immunoreactive bands were detected with a chemiluminescence kit (Millipore, Billerica, MA, USA) according to the manufacturer's procedure.

Cell proliferation assay. Cell Titer 96 non-radioactive cell proliferation (MTS) (Promega BioSciences, Madison, WI, USA) and Cell-Light ${ }^{\mathrm{TM}}$ EdU cell proliferation detection (EdU) assays (RiboBio Co., Ltd.) were performed to test the proliferation ability of the cells following the manufacturer's protocol. Briefly, for the MTS assay, transiently transfected cells were planted in 96-well plates with 6,000 cells/well and incubated for 24,48 and $96 \mathrm{~h}$. The viability of the cells was determined with MTS. The absorbance value at a wavelength of $490 \mathrm{~nm}$ was used as an indicator of cell viability. For the EdU assay, $24 \mathrm{~h}$ after transfection, the cells were cultured in triplicate at 6,000 cells/well in 96-well plates the day before EdU incubation. After EdU labeling, the cells were treated with $100 \mu \mathrm{l}$ of Apollo reaction cocktail. Then nucleic acids in all cells were stained with 4',6-diamidino-2-phenylindole (DAPI) and visualized under a fluorescence microscope (Olympus, Tokyo, Japan). The percentage of EdU-positive cells was defined as the proliferation rate. Data were obtained from three independent experiments.

Cell migration and invasion assays. Migration assays were performed with Transwell inserts containing a polycarbonate membrane with $8.0 \mu \mathrm{m}$ pores (Corning, New York, NY, USA). To measure the invasion of cancer cells, membranes were coated with Matrigel matrix (BD Biosciences, Bedford, MA, USA). Forty-eight hours after transfection with the siRNA or the negative control, $1 \times 10^{5}$ cells in $200 \mu \mathrm{l}$ serum-free media were added into the inside chamber and $600 \mu \mathrm{l}$ medium with $10 \% \mathrm{FBS}$ was added to the outside chamber. After incubated for $24 \mathrm{~h}$ at $37^{\circ} \mathrm{C}$ in a $\mathrm{CO}_{2}$ incubator, cells on the inner surface were removed softly, while the migrated or invaded cells that attached to the bottom of the membrane insert were fixed and stained with $1 \%$ crystal violet. Then the cells were counted under a microscope in 5 different fields.

Statistical analysis. All statistical analyses were performed using SPSS 19.0 software (SPSS, Inc., Chicago, IL, USA). A Student's t-test was used to analyze difference between two groups. The Chi-square test and Fisher's exact test were used to analyze the relationship between TMPRSS4 expression and the clinicopathological parameters. OS and DFS were evaluated with the Kaplan-Meier method, and differences were compared by log-rank test. Analyses of predictive factors for OS and DFS were performed with univariate and multivariate Cox proportional hazards regression method. Data represent the means \pm standard deviation $(\mathrm{SD})$ from 3 independent experiments. A two-tailed value of $\mathrm{P}<0.05$ was considered statistically significant.
Table I. Association of TMPRSS4 expression with clinicopathological features of the patients with BC.

\begin{tabular}{|c|c|c|c|c|}
\hline \multirow[b]{2}{*}{ Variables } & \multirow[b]{2}{*}{ No. $(n=107)$} & \multicolumn{2}{|c|}{$\begin{array}{l}\text { TMPRSS4 } \\
\text { expression }\end{array}$} & \multirow[b]{2}{*}{ P-value } \\
\hline & & Low & High & \\
\hline Age (years) & & & & 0.474 \\
\hline$\leq 50$ & 47 & 18 & 29 & \\
\hline$>50$ & 60 & 19 & 41 & \\
\hline Menopausal status & & & & 0.158 \\
\hline Premenopausal & 58 & 20 & 38 & \\
\hline Postmenopausal & 49 & 17 & 32 & \\
\hline Tumor size (cm) & & & & 0.044 \\
\hline$\leq 2$ & 41 & 19 & 22 & \\
\hline$>2$ & 66 & 18 & 48 & \\
\hline Histological subtype & & & & 0.717 \\
\hline Ductal & 70 & 22 & 48 & \\
\hline Lobular & 13 & 6 & 7 & \\
\hline Mucinous & 12 & 4 & 8 & \\
\hline Micropapillary & 12 & 5 & 7 & \\
\hline Grade of ductal cancer & & & & 0.006 \\
\hline I & 16 & 9 & 7 & \\
\hline II & 35 & 10 & 25 & \\
\hline III & 19 & 3 & 16 & \\
\hline $\mathrm{LN}$ metastasis & & & & 0.002 \\
\hline Negative & 59 & 28 & 31 & \\
\hline Positive & 48 & 9 & 39 & \\
\hline Clinical stage & & & & 0.015 \\
\hline I, II & 58 & 26 & 32 & \\
\hline III, IV & 49 & 11 & 38 & \\
\hline ER status ${ }^{\mathrm{a}}$ & & & & 0.292 \\
\hline Negative & 45 & 13 & 32 & \\
\hline Positive & 62 & 24 & 38 & \\
\hline PR status ${ }^{\mathrm{a}}$ & & & & 0.428 \\
\hline Negative & 49 & 15 & 34 & \\
\hline Positive & 58 & 22 & 36 & \\
\hline HER2 status ${ }^{\mathrm{a}}$ & & & & 0.362 \\
\hline Negative (IHC 0-2+) & 67 & 21 & 46 & \\
\hline Positive (IHC 3+) & 40 & 16 & 24 & \\
\hline
\end{tabular}

${ }^{a}$ ER, PR and HER2 statuses were determined by immunohistochemical staining according to a previously defined guideline (18). BC, breast cancer; LN, lymph node; ER, estrogen receptor; PR, progesterone receptor; HER2, human epidermal growth factor receptor-2.

\section{Results}

TMPRSS4 is overexpressed in breast cancer tissues. The characteristics of the study population are summarized in Table I. Patient age ranged from 25 to 78 years, with a mean age of 51.6 years. Median follow-up time was 65.2 months. TMPRSS4-positive staining was located mainly in the 


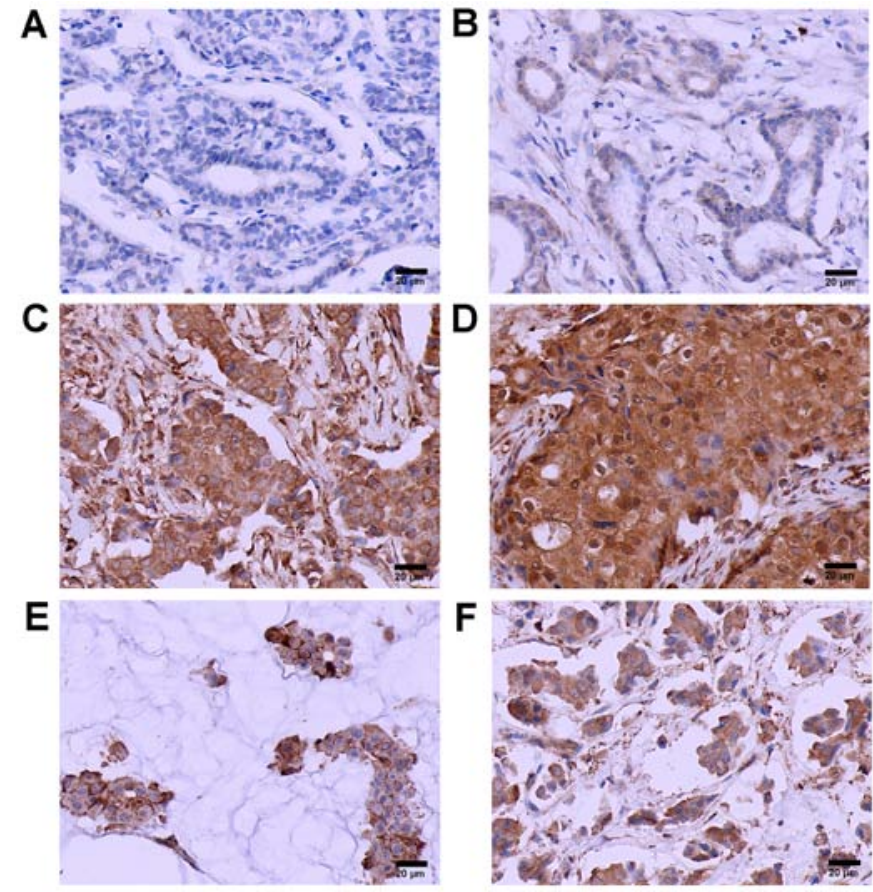

Figure 1. Transmembrane protease serine 4 (TMPRSS4) is overexpressed in breast cancer (BC) tissues. Immunohistochemical staining of TMPRSS4 (in brown) in: (A) normal breast tissues, (B) grade I BC tissues, (C) grade II BC tissues, (D) grade III BC tissues, (E) invasive mucinous carcinoma tissues and $(\mathrm{F})$ invasive micropapillary carcinoma tissues (original magnification, all $\mathrm{x} 400)$. Scale bar, $20 \mu \mathrm{m}$.

cytoplasm or cell membrane of the tumor cell nests. The positive TMPRSS4 expression rate was $65.4 \%(70 / 107)$ in BC cases and $17.6 \%$ (9/52) in normal breast tissues (Fig. 1). A significant statistical difference was found between the two groups $(\mathrm{P}<0.05)$. Furthermore, the expression of TMPRSS4 was increased along with grade, with the highest expression in grade III BC tissues and the lowest expression in grade I $\mathrm{BC}$ tissues $(\mathrm{P}<0.05)$. TMPRSS4 was also overexpressed in invasive mucinous carcinoma and micropapillary carcinoma tissues (Fig. 1E and F) with no statistically significant differences between them.

Correlation of TMPRSS4 with different clinicopathological parameters. Next, we evaluated the associations between TMPRSS4 protein expression and a series of clinicopathological characteristics including age, menopausal status, tumor size, histological type, histological grade, lymph node stage, clinical stage, and status of ER, PR and HER2 in the BC patients. As shown in Table I, high expression of TMPRSS4 was positively correlated with tumor size $(\mathrm{P}=0.044)$, histological grade $(\mathrm{P}=0.006)$, lymph node metastasis $(\mathrm{P}=0.002)$, clinical stage $(\mathrm{P}=0.015)$, but was not correlated with other clinicopathological parameters, including patient age $(\mathrm{P}=0.474)$, menopausal status $(\mathrm{P}=0.158)$, histological subtype $(\mathrm{P}=0.717)$, and status of ER $(\mathrm{P}=0.292), \mathrm{PR}(\mathrm{P}=0.428)$ and HER2 ( $\mathrm{P}=0.362)$.

Overexpression of TMPRSS4 is correlated with poor outcomes. We then investigated whether the expression of TMPRSS4 is associated with clinical outcomes in BC. The OS and DFS
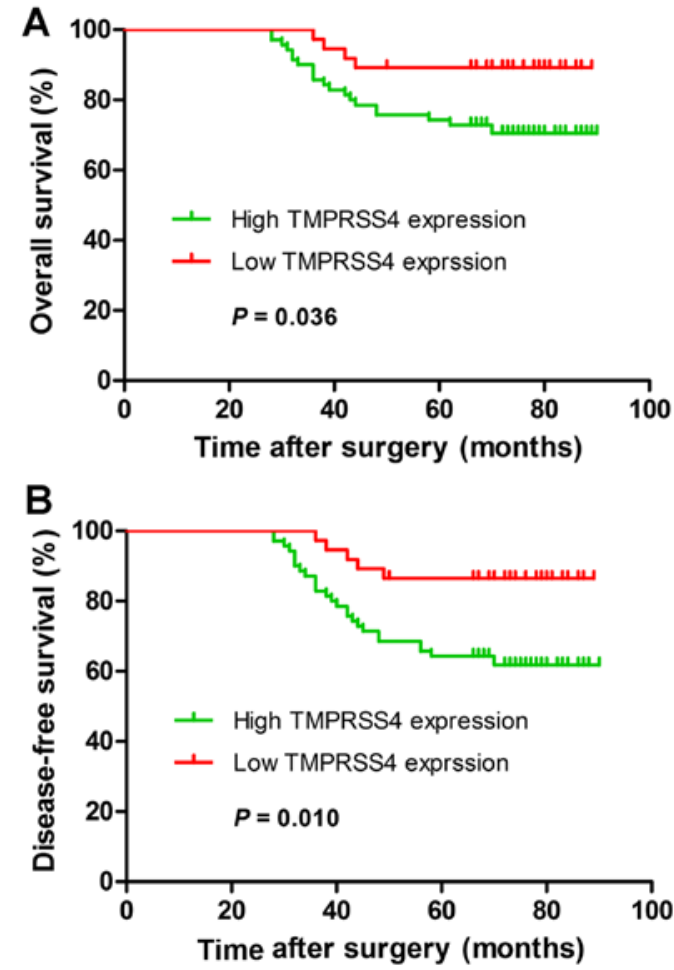

Figure 2. Kaplan-Meier survival curves for breast cancer (BC) patients stratified according to transmembrane protease serine 4 (TMPRSS4) expression. (A) Overall survival (OS) curves of BC patients according to TMPRSS4 immunostaining. (B) Disease-free survival (DFS) curves of BC patients according to TMPRSS4 immunostaining. P-values were obtained by logrank test.

indicated by the Kaplan-Meier survival curves of the BC patients according to low and high TMPRSS4 expression are shown in Fig. 2. Patients with high expression of TMPRSS4 had shorter OS $(\mathrm{P}=0.036)$ and DFS $(\mathrm{P}=0.010)$ when compared with the patients with low TMPRSS4 expression. Univariate and multivariate analyses were carried out using Cox proportional hazard model to evaluate the influence of TMPRSS4 expression and pathological factors on the prognosis of $\mathrm{BC}$ patients. In Table II, the univariate analysis revealed that tumor size (OS, $\mathrm{P}=0.042$; DFS, $\mathrm{P}=0.047)$, lymph node metastasis (OS, $\mathrm{P}=0.000$; DFS, $\mathrm{P}=0.001)$, histological grade $(\mathrm{OS}, \mathrm{P}=0.036$; DFS, $\mathrm{P}=0.039)$, clinical stage $(\mathrm{OS}, \mathrm{P}=0.009$; $\mathrm{DFS}, \mathrm{P}=0.012)$, and TMPRSS4 expression (OS, $\mathrm{P}=0.015$; DFS, $\mathrm{P}=0.018$ ) were prognostic factors for $\mathrm{BC}$ patients. The multivariate analysis indicated that TMPRSS4 expression was one of the independent prognostic factors, along with clinical stage and lymph node metastasis (Table II).

TMPRSS4 knockdown suppresses the proliferation of BC cells in vitro. As TMPRSS4 was highly expressed in BC tissues, we further detected the expression level of TMPRSS4 in different BC cell lines. RT-qPCR and western blot analysis showed that TMPRSS4 was differentially expressed in five BC cell lines, with the highest expression in MDA-MB-468 cells (Fig. 3A).

To inhibit endogenous high TMPRSS4 levels, two cell lines (MDA-MB-468 and MDA-MB-231) were transfected with a small interfering RNA (siRNA) duplex and transfection 
Table II. Univariate and multivariate Cox regression analyses of overall survival and disease-free survival in patients with BC.

\begin{tabular}{|c|c|c|c|c|c|c|}
\hline \multirow[b]{2}{*}{ Variables } & \multicolumn{3}{|c|}{ Univariate analysis } & \multicolumn{3}{|c|}{ Multivariate analysis } \\
\hline & HR & $95 \% \mathrm{CI}$ & P-value & HR & $95 \% \mathrm{CI}$ & P-value \\
\hline \multicolumn{7}{|l|}{ OS } \\
\hline Age ( $\leq 50$ vs. $>50$ years $)$ & 1.362 & $1.282-2.530$ & 0.533 & & & \\
\hline Menopausal status (pre vs. post) & 1.342 & $0.601-2.993$ & 0.473 & & & \\
\hline Histological subtype (ductal vs. lobular) & 1.087 & $0.932-1.397$ & 0.710 & & & \\
\hline Tumor size ( $\leq 2$ vs. $>2 \mathrm{~cm})$ & 1.549 & $1.247-1.222$ & 0.042 & 2.168 & $0.724-2.854$ & 0.068 \\
\hline Grade of ductal cancer (I, II vs. III) & 2.372 & $1.532-3.278$ & 0.036 & 2.874 & $1.153-3.238$ & 0.057 \\
\hline LN metastasis (negative vs. positive) & 1.114 & $1.034-2.382$ & 0.000 & 1.136 & $1.040-1.460$ & 0.001 \\
\hline Clinical stage (I, II vs. III, IV) & 2.166 & $1.420-3.218$ & 0.009 & 2.039 & $1.027-3.995$ & 0.015 \\
\hline ER status (negative vs. positive) & 1.458 & $1.032-2.688$ & 0.297 & & & \\
\hline PR status (negative vs. positive) & 1.362 & $1.067-2.876$ & 0.374 & & & \\
\hline HER2 status (negative vs. positive) & 1.299 & $0.998-1.745$ & 0.107 & & & \\
\hline TMPRSS4 expression (low vs. high) & 1.265 & $1.091-1.777$ & 0.015 & 1.289 & $1.098-1.850$ & 0.016 \\
\hline \multicolumn{7}{|l|}{ DFS } \\
\hline Age $(\leq 50$ vs. $>50$ years $)$ & 1.562 & $1.187-2.623$ & 0.624 & & & \\
\hline Menopausal status (pre vs. post) & 1.447 & $0.648-3.232$ & 0.367 & & & \\
\hline Histological subtype (ductal vs. lobular) & 1.396 & $0.993-2.056$ & 0.802 & & & \\
\hline Tumor size ( $\leq 2 \mathrm{vs} .>2 \mathrm{~cm})$ & 1.567 & $1.278-1.793$ & 0.047 & 2.272 & $0.927-3.568$ & 0.073 \\
\hline Grade of ductal cancer (I, II vs. III) & 2.569 & $1.587-2.763$ & 0.039 & 2.942 & $1.278-3.524$ & 0.066 \\
\hline LN metastasis (negative vs. positive) & 2.054 & $1.029-3.398$ & 0.001 & 2.143 & $1.042-3.489$ & 0.002 \\
\hline Clinical stage (I, II vs. III, IV) & 3.971 & $1.355-5.637$ & 0.012 & 2.873 & $1.964-5.761$ & 0.018 \\
\hline ER status (negative vs. positive) & 1.545 & $1.058-2.734$ & 0.301 & & & \\
\hline PR status (negative vs. positive) & 1.378 & $1.075-2.888$ & 0.381 & & & \\
\hline HER2 status (negative vs. positive) & 1.282 & $0.989-1.763$ & 0.147 & & & \\
\hline TMPRSS4 expression (low vs. high) & 1.278 & $1.137-1.821$ & 0.018 & 1.355 & $1.185-1.971$ & 0.019 \\
\hline
\end{tabular}

BC, breast cancer; OS, overall survival; DFS, disease-free survival; HR, hazard ratio; CI, confidence interval; LN, lymph node; ER, estrogen receptor; PR, progesterone receptor; HER2, human epidermal growth factor receptor-2.

efficiency was evaluated by RT-qPCR. Substantial knockdown of TMPRSS4 mRNA was observed in the MDA-MB-468 and MDA-MB-231 cells (all $\mathrm{P}<0.001$ ) (Fig. 3B). Following siRNA transfection, the cell proliferation rate was evaluated by MTS and EdU assays. MTS assay showed that the cell proliferation rate was significantly decreased in both the MDA-MB-468 and MDA-MB-231 cell lines $96 \mathrm{~h}$ after transfection (all $\mathrm{P}<0.05$ ) (Fig. 3C and D). Similar changes were further observed in the EdU assay (Fig. 4A and C). The cell proliferation rate was significantly decreased by $42.97 \pm 3.04$ and $27.53 \pm 4.60 \%$ in the MDA-MB-468 and MDA-MB-231 cells (all $\mathrm{P}<0.01)$ (Fig. 4B and D).

TMPRSS4 knockdown inhibits the migration and invasion abilities of BC cells in vitro. Given that TMPRSS4 was highly expressed in metastatic breast cancer tissues and cell lines, the roles of TMPRSS4 in cell migration and invasion in breast cancer were also investigated. As shown in Fig. 5A and C, MDA-MB-468 and MDA-MB-231 cells transfected with siRNA showed a significantly decreased migration and invasion capability, compared with the untreated cells. Transfected cells showed a considerable decrease in migration activity by $46.40 \pm 1.68$ and $50.12 \pm 2.41 \%$ and a decrease in invasion capacity by $45.59 \pm 2.75$ and $50.13 \pm 2.49 \%$ in the MDA-MB-468 (all $\mathrm{P}<0.001)$ (Fig. 5B) and MDA-MB-231 $(\mathrm{P}<0.05$ and $\mathrm{P}<0.001)$ (Fig. 5D) cells, respectively, when compared with these abilities noted in the negative control group. Collectively, these results suggest that knockdown of TMPRSS4 significantly inhibited the migration and invasion activity of BC cells in vitro.

TMPRSS4 knockdown influences the expression of EMTrelated genes. Following siRNA transfection, TMPRSS4 protein expression was determined in the MDA-MB-468 and MDA-MB-231 cells by western blot analysis (all $\mathrm{P}<0.05$ ) (Fig. 6C and D). To verify the relationship between TMPRSS4 expression and EMT, we observed the expression of key biomarkers of EMT after TMPRSS4 was silenced. RT-qPCR and western blot analysis revealed that TMPRSS4 knockdown significantly enhanced the expression of E-cadherin and claudin-1 and inhibited the expression of vimentin and Slug in the MDA-MB-468 cells (all $\mathrm{P}<0.05$ ) (Fig. 6A, C and E), indicating suppression of EMT. Similar results were observed in the MDA-MB-231 cells (all $\mathrm{P}<0.05$ ) (Fig. 6B, D and F). The expression of ZEB1 did not show a significantly different change $(\mathrm{P}>0.05)$, and the expression of Snail was undetectable in the two cell lines (data not shown). 
A
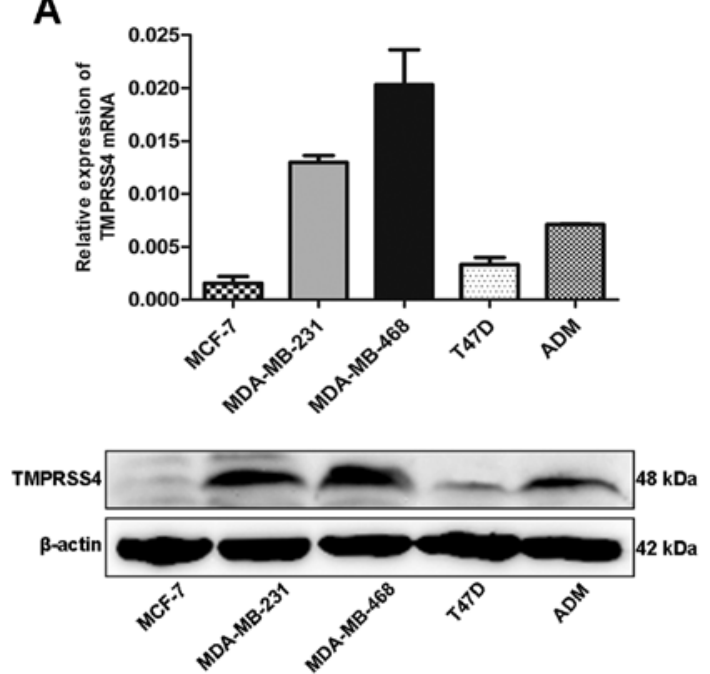

C

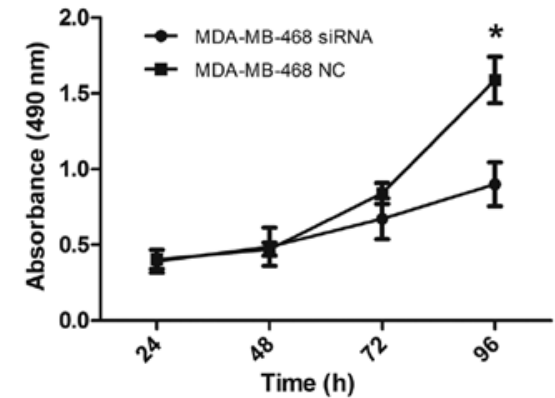

B
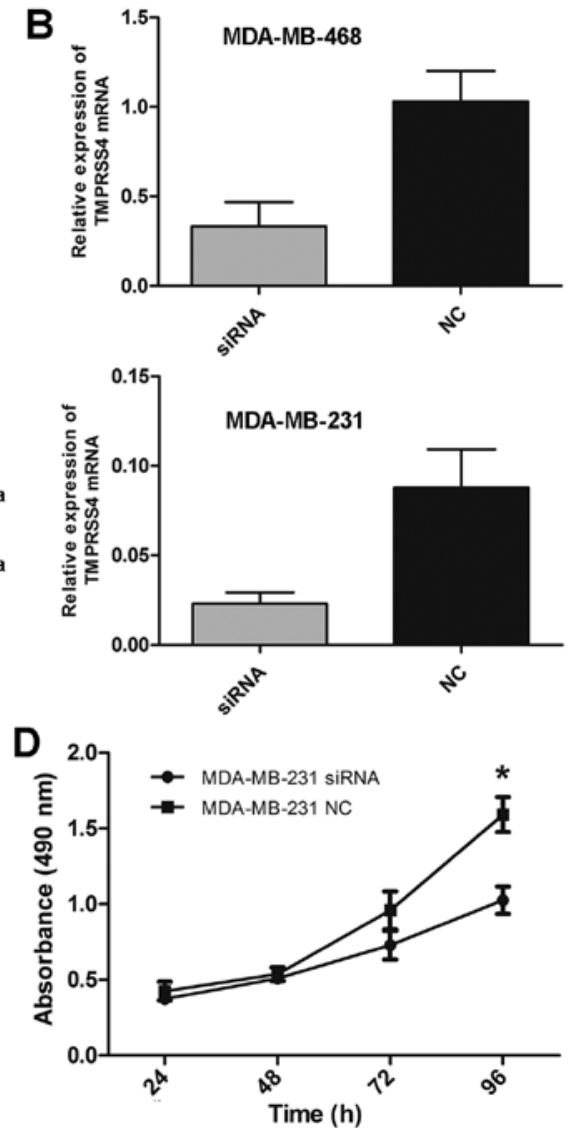

Figure 3. Knockdown of transmembrane protease serine 4 (TMPRSS4) suppresses cell proliferation. (A) mRNA (upper) and protein (lower) expression of TMPRSS4 in different breast cancer (BC) cell lines. (B) siRNA transfection significantly reduced TMPRSS4 mRNA expression in MDA-MB-468 and MDA-MB-231 cells. (C and D) Proliferation rates of MDA-MB-468 and MDA-MB-231 cells were reduced after TMPRSS4 knockdown. " $\mathrm{P}<0.05$. NC, negative control.

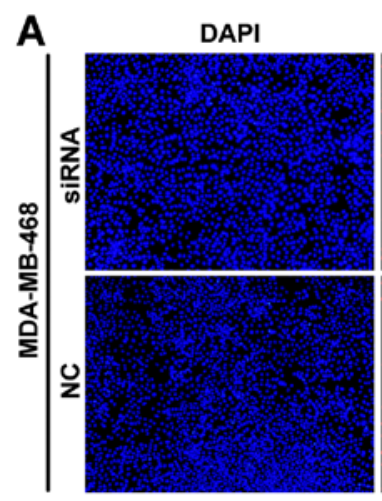

C

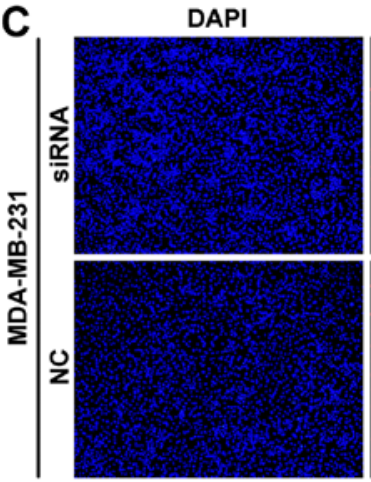

EdU

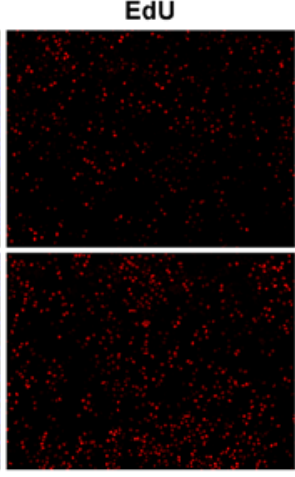

EdU

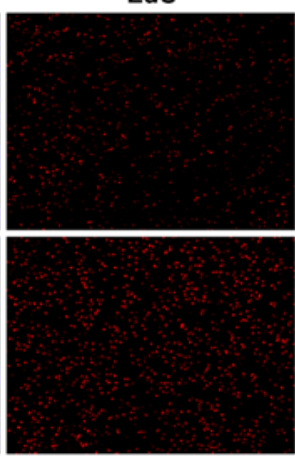

Merged

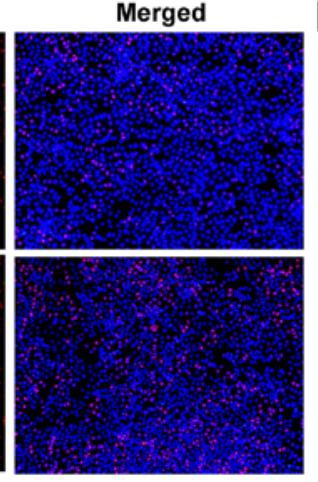

Merged

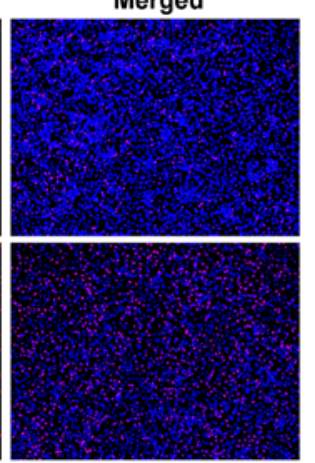

B

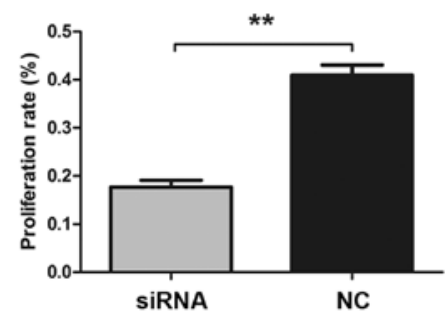

D

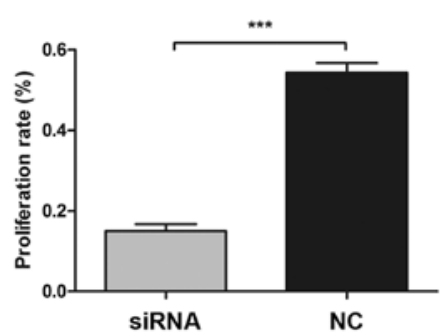

Figure 4. Effects of transmembrane protease serine 4 (TMPRSS4) knockdown on cell proliferation in MDA-MB-468 and MDA-MB-231 cell lines using EdU assay. (A and C) Representative images after transfection with siRNA in MDA-MB-468 and MDA-MB-231 cells (magnification, x100). (B and D) The percentage of EdU-positive cells, calculated by the EdU-labeled cell (red) number compared to total cell number (DAPI, blue), was defined as the proliferation rate. Cells with siRNA transfection showed significantly fewer proliferating cells than the negative control group. ${ }^{* *} \mathrm{P}<0.01$ and ${ }^{* * *} \mathrm{P}<0.001$. NC, negative control 
A

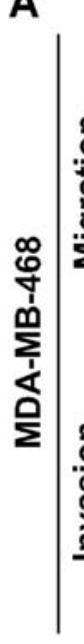

C

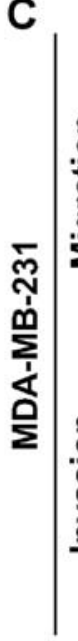

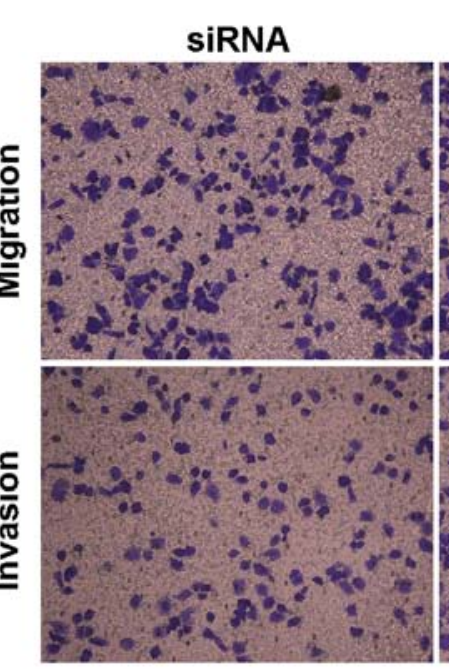

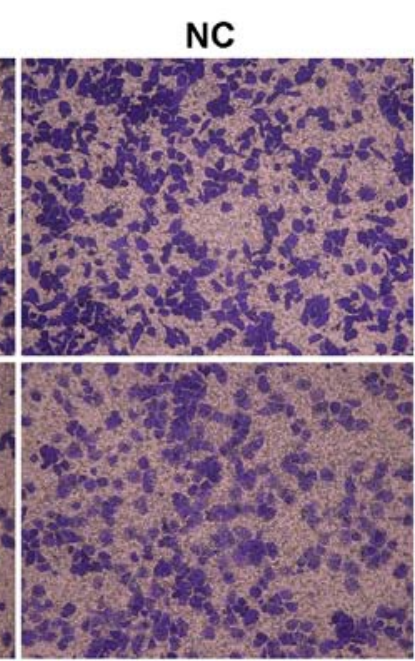

B
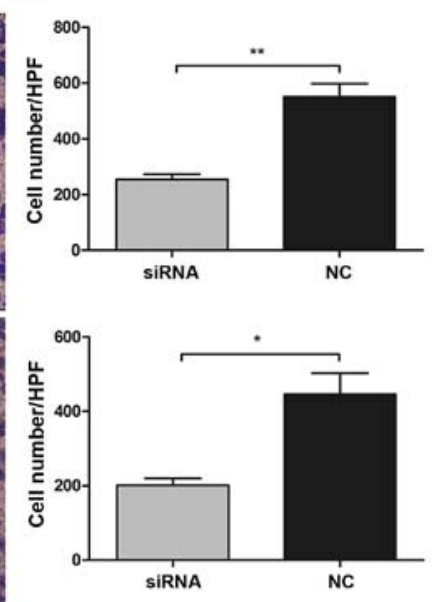

D

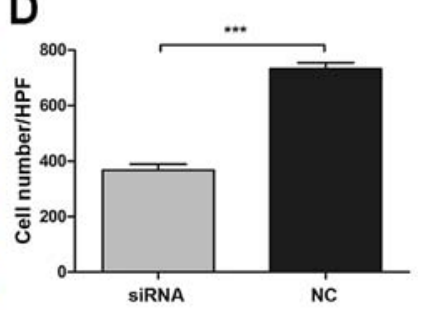

NC

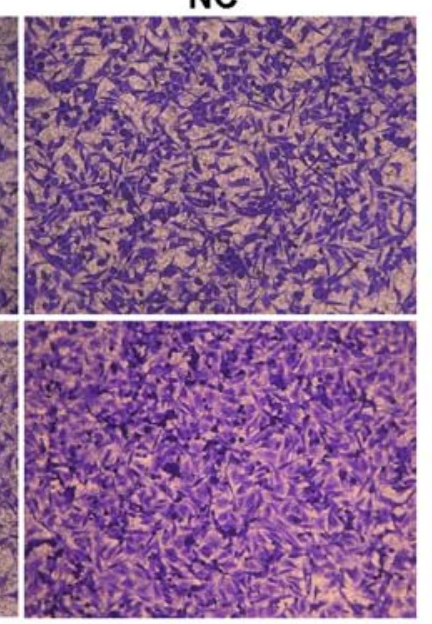

SiRNA

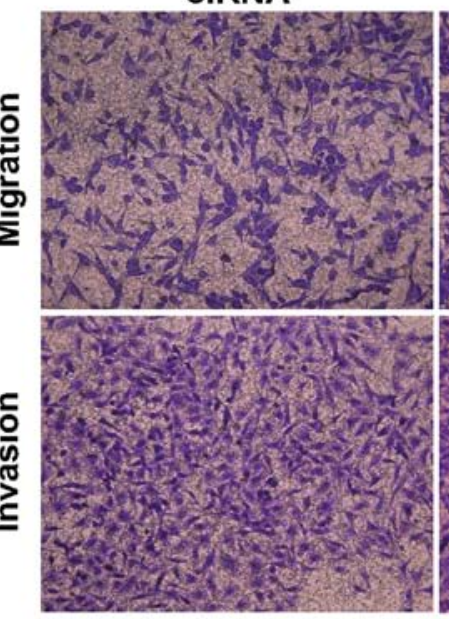

Figure 5. Influence of transmembrane protease serine 4 (TMPRSS4) knockdown on cell migration and invasion in MDA-MB-468 and MDA-MB-231 cells. (A and C) Representative images of migration and invasion assays in the MDA-MB-468 and MDA-MB-231 cells (magnification, $\mathrm{x} 200$ ) are shown. (B and D) The migration and invasion abilities of the MDA-MB-468 and MDA-MB-231 were decreased in the cells transfected with siRNA in comparison with the negative control. ${ }^{*} \mathrm{P}<0.05,{ }^{* *} \mathrm{P}<0.01$ and ${ }^{* * *} \mathrm{P}<0.001 . \mathrm{NC}$, negative control.

\section{Discussion}

The carcinogenesis and development of breast cancer (BC) is linked to different molecular events, and the lack of effective markers for the prediction of prognosis makes it difficult to apply individualized treatment protocols to BC patients. Proteases have been extensively studied as important participants in the carcinogenesis of many types of tumors (6-8). Recently, much attention has focused on the role of TTSPs during tumor development and progression $(11,19)$. As a member of the family of cell surface-associated proteases, TTSPs modulate a variety of normal cellular activities as well as tumor invasion and metastasis $(19,20)$.

TMPRSS4, initially referred to as TMPRSS3, is located on chromosome 11.q23.3 and encodes a member of the type II TTSP family (21). Previous studies have shown that TMPRSS4 is highly expressed in different types of cancer, such as pancreatic cancer (21), thyroid cancer (22), lung cancer (23) and hepatocellular carcinoma (15). Overexpression of TMPRSS4 in non-small cell lung cancer is associated with poor prognosis in patients with squamous histology (24). Huang et al (25) reported that TMPRSS4 is highly expressed in colorectal cancer tissues both at the mRNA and protein level and is correlated with pathological stage. In BC and triple-negative BC (TNBC), high expression of TMPRSS4 was found to be indicative of poor prognosis and related to tumor size, $\mathrm{LN}$ metastasis and histological grade $(26,27)$. Hence, TMPRSS4 appears to be a factor regulating tumor development and progression. The present study confirmed that TMPRSS4 was highly expressed in BC tissues compared with normal breast tissues, and we further explored the relationship between TMPRSS4 expression and histological subtypes. Notably, TMPRSS4 was not only overexpressed in invasive ductal carcinoma but also in invasive mucinous carcinoma and micropapillary carcinoma. In addition, expression of TMPRSS4 was increased along with grade, with the highest expression in grade III BC tissues and the lowest in grade I BC tissues, suggesting a positive correlation between TMPRSS4 and BC progression.

Our results also showed that TMPRSS4 overexpression is significantly correlated with tumor size, histological grade, lymph node metastasis, and clinical stage, suggesting that TMPRSS4 may be involved in the progression of BC. Survival 

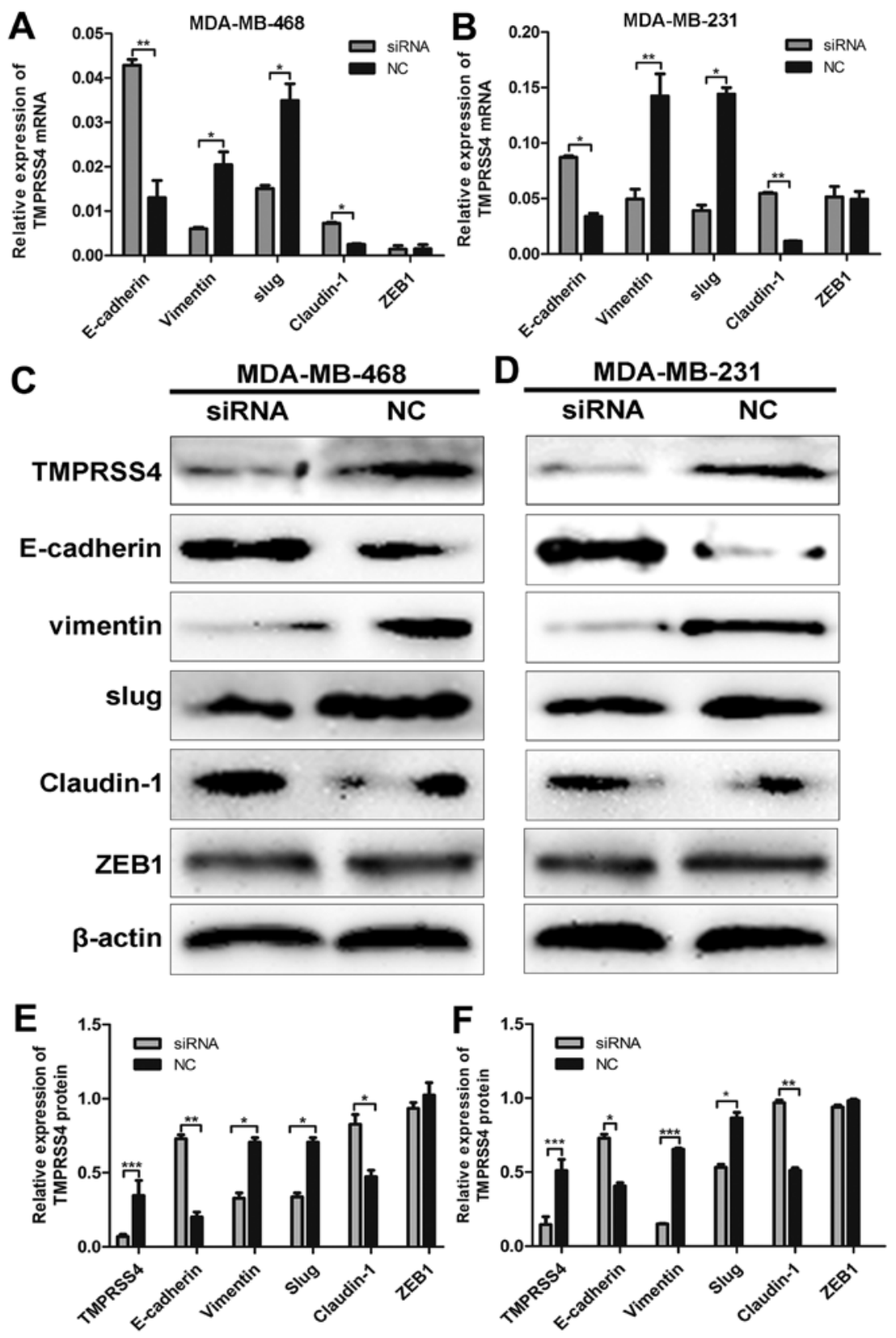

Figure 6. Knockdown of transmembrane protease serine 4 (TMPRSS4) regulated the expression of epithelial-mesenchymal transition (EMT) related genes. (A and B) RT-qPCR demonstrated that EMT markers of E-cadherin and vimentin were upregulated and downregulated respectively after TMPRSS4 knockdown in MDA-MB-468 and MDA-MB-231 cells. For the expression of key EMT transcriptional factors, claudin-1 and slug were upregulated and downregulated after transfection with siRNA, respectively. However, expression of ZEB1 did not show significant change. (C-F) Transfection efficiency and similar results were further observed in western blot analysis. $\beta$-actin was an internal control. ${ }^{*} \mathrm{P}<0.05,{ }^{* * *} \mathrm{P}<0.01$ and ${ }^{* * * *} \mathrm{P}<0.001$. NC, negative control; EMT, epithelial-mesenchymal transition.

analysis demonstrated that high TMPRSS4 expression is associated with shorter DFS and OS in BC patients. Univariate and multivariate Cox regression analyses revealed that TMPRSS4 could serve as an independent prognostic factor for BC. All these results confirmed that TMPRSS4 is an important predictive indicator of $\mathrm{BC}$ prognosis.

We identified the overexpression of TMPRSS4 in BC tissues and different histological subtypes. Furthermore, RT-qPCR and western blot analysis demonstrated that TMPRSS4 was differentially expressed in five BC cell lines, with the highest expression in MDA-MB-468 cells. Then silencing of TMPRSS4 in MDA-MB-468 and MDA-MB-231 cells significantly inhibited cell proliferation, migration and invasion in vitro. Here, we show initial evidence that TMPRSS4 is highly expressed in BC cell lines. However, the biological functions of TMPRSS4 and its potential mechanisms in BC cells are not well understood. A recent study reported that TFPI-2 negatively regulated cell growth by inhibiting transcription of TMPRSS4 (28). Kim et al (14) also reported that TMPRSS4 induces invasion, migration, and metastasis of cancer cells by facilitating EMT events; TMPRSS4 induces invasion and EMT through upregulation of integrin $\alpha 5$ and its signaling pathways.

EMT is a process implicated in the conversion of early stage tumors to invasive malignancies. Induction of EMT allows tumor cells to metastasize and establish secondary tumors at 
a distant site due to weak intercellular adhesion and enhanced cell motility $(13,29,30)$. Loss of E-cadherin transcript, a hallmark of EMT, plays a central role in the EMT process (13). In an attempt to determine the mechanism by which E-cadherin is downregulated, we examined several well-known E-cadherin transcriptional repressors/EMT-inducing transcriptional repressors, including Slug, Snail, SIP1/ZEB1 and E12/E47 (31). In this study, western blot analysis showed that E-cadherin and claudin-1 were upregulated after TMPRSS4 knockdown. On the contrary, Slug and vimentin were downregulated, indicating that TMPRSS4 contributes to tumor cell invasion and metastasis by promoting EMT. Cheng et al (15) suggested that TMPRSS4-induced EMT was mediated through Snail and Slug as a result of Raf/MEK/ERK1/2 activation. However, the specific regulatory mechanisms were not elucidated in our results and further in-depth studies are required to validate these signaling pathways in BC cells.

Taken together, our results demonstrated that the upregulation of TMPRSS4 expression is a key event in BC progression and it could promote cell proliferation, migration and invasion abilities through possible induction of EMT. TMPRSS4 could be regarded as a potential prognostic biomarker and a therapeutic target for $\mathrm{BC}$.

\section{Acknowledgements}

This study was supported by the National Natural Science Foundation of China (no. 81372856) and Taishan Scholars Program of Shandong Province (no. ts201511096).

\section{References}

1. Miao H, Hartman M, Bhoo-Pathy N, Lee SC, Taib NA, Tan EY, Chan P, Moons KG, Wong HS, Goh J, et al: Predicting survival of de novo metastatic breast cancer in Asian women: Systematic review and validation study. PLoS One 9: e93755, 2014.

2. Cummings MC, Chambers R, Simpson PT and Lakhani SR: Molecular classification of breast cancer: Is it time to pack up our microscopes? Pathology 43: 1-8, 2011

3. Ginsburg GS and Willard HF: Genomic and personalized medicine: Foundations and applications. Transl Res 154: 277-287, 2009.

4. Dechaphunkul A, Phukaoloun M, Kanjanapradit K, Graham K, Ghosh S, Santos C and Mackey JR: Prognostic significance of tissue inhibitor of metalloproteinase-1 in breast cancer. Int J Breast Cancer 2012: 290854, 2012.

5. Geyer FC, Rodrigues DN, Weigelt B and Reis-Filho JS: Molecular classification of estrogen receptor-positive/luminal breast cancers. Adv Anat Pathol 19: 39-53, 2012.

6. Duffy MJ: Proteases as prognostic markers in cancer. Clin Cancer Res 2: 613-618, 1996.

7. Roy R, Yang J and Moses MA: Matrix metalloproteinases as novel biomarkers and potential therapeutic targets in human cancer. J Clin Oncol 27: 5287-5297, 2009.

8. Flores-Reséndiz D, Castellanos-Juárez E and BenítezBribiesca L: Proteases in cancer progression. Gac Med Mex 145: 131-142, 2009 (In Spanish).

9. Netzel-Arnett S, Hooper JD, Szabo R, Madison EL, Quigley JP, Bugge TH and Antalis TM: Membrane anchored serine proteases: A rapidly expanding group of cell surface proteolytic enzymes with potential roles in cancer. Cancer Metastasis Rev 22: 237-258, 2003.

10. Hooper JD, Clements JA, Quigley JP and Antalis TM: Type II transmembrane serine proteases. Insights into an emerging class of cell surface proteolytic enzymes. J Biol Chem 276: 857-860, 2001.

11. Choi SY, Bertram S, Glowacka I, Park YW and Pöhlmann S Type II transmembrane serine proteases in cancer and viral infections. Trends Mol Med 15: 303-312, 2009.

12. de Aberasturi AL and Calvo A: TMPRSS4: An emerging potential therapeutic target in cancer. Br J Cancer 112: 4-8, 2015.
13. Jung H, Lee KP, Park SJ, Park JH, Jang YS, Choi SY, Jung JG, Jo K, Park DY, Yoon JH, et al: TMPRSS4 promotes invasion, migration and metastasis of human tumor cells by facilitating an epithelial-mesenchymal transition. Oncogene 27: 2635-2647, 2008.

14. Kim S, Kang HY, Nam EH, Choi MS, Zhao XF, Hong CS, Lee JW, Lee JH and Park YK: TMPRSS4 induces invasion and epithelial-mesenchymal transition through upregulation of integrin alpha5 and its signaling pathways. Carcinogenesis 31: 597-606, 2010.

15. Wang CH, Guo ZY, Chen ZT, Zhi XT, Li DK, Dong ZR, Chen ZQ, Hu SY and Li T: TMPRSS4 facilitates epithelial-mesenchymal transition of hepatocellular carcinoma and is a predictive marker for poor prognosis of patients after curative resection. Sci Rep 5: 12366, 2015.

16. Edge SB, Byrd DR, Compton CC, Fritz AG, Greene FL and Trotti A (eds): AJCC Cancer Staging Manual. 7th edition. Springer, New York, NY, pp237-246, 2010.

17. Lakhani SR, Ellis IO, Schnitt SJ, Tan PH and van de Vijver MJ (eds): WHO classification of tumours of the breast. World Health Organization classification of tumours. 4th edition. IARC Press, Lyon, 2012.

18. Wolff AC, Hammond ME, Hicks DG, Dowsett M, McShane LM, Allison KH, Allred DC, Bartlett JM, Bilous M, Fitzgibbons P, et al; American Society of Clinical Oncology; College of American Pathologists: Recommendations for human epidermal growth factor receptor 2 testing in breast cancer: American Society of Clinical Oncology/College of American Pathologists clinical practice guideline update. J Clin Oncol 31: 3997-4013, 2013.

19. Szabo R and Bugge TH: Type II transmembrane serine proteases in development and disease. Int $\mathrm{J}$ Biochem Cell Biol 40: 1297-1316, 2008.

20. Antalis TM, Bugge TH and Wu Q: Membrane-anchored serine proteases in health and disease. Prog Mol Biol Transl Sci 99: $1-50,2011$.

21. Wallrapp C,Hähnel S, Müller-Pillasch F, Burghardt B, Iwamura T, Ruthenbürger M, Lerch MM, Adler G and Gress TM: A novel transmembrane serine protease (TMPRSS3) overexpressed in pancreatic cancer. Cancer Res 60: 2602-2606, 2000.

22. Kebebew E, Peng M, Reiff E, Duh QY, Clark OH and McMillan A: ECM1 and TMPRSS4 are diagnostic markers of malignant thyroid neoplasms and improve the accuracy of fine needle aspiration biopsy. Ann Surg 242: 353-363, 2005.

23. Nguyen TH, Weber W, Havari E, Connors T, Bagley RG, McLaren R, Nambiar PR, Madden SL, Teicher BA, Roberts B, et al: Expression of TMPRSS4 in non-small cell lung cancer and its modulation by hypoxia. Int J Oncol 41: 829-838, 2012.

24. Larzabal L, Nguewa PA,Pio R, Blanco D, Sanchez B, Rodríguez MJ, Pajares MJ, Catena R, Montuenga LM and Calvo A: Overexpression of TMPRSS4 in non-small cell lung cancer is associated with poor prognosis in patients with squamous histology. Br J Cancer 105: 1608-1614, 2011.

25. Huang A, Zhou H, Zhao H, Quan Y, Feng B and Zheng M: TMPRSS4 correlates with colorectal cancer pathological stage and regulates cell proliferation and self-renewal ability. Cancer Biol Ther 15: 297-304, 2014.

26. Liang B, Wu M, Bu Y, Zhao A and Xie F: Prognostic value of TMPRSS4 expression in patients with breast cancer. Med Oncol 30: 497, 2013.

27. Cheng D, Kong $\mathrm{H}$ and Li Y: TMPRSS4 as a poor prognostic factor for triple-negative breast cancer. Int J Mol Sci 14: 14659-14668, 2013.

28. Hamamoto J, Soejima K, Naoki K, Yasuda H, Hayashi Y, Yoda S, Nakayama S, Satomi R, Terai H, Ikemura S, et al: Methylationinduced downregulation of TFPI-2 causes TMPRSS4 overexpression and contributes to oncogenesis in a subset of nonsmall-cell lung carcinoma. Cancer Sci 106: 34-42, 2015.

29. Brockhausen J, Tay SS, Grzelak CA, Bertolino P, Bowen DG, d'Avigdor WM, Teoh N, Pok S, Shackel N, Gamble JR, et al: miR-181a mediates TGF- $\beta$-induced hepatocyte EMT and is dysregulated in cirrhosis and hepatocellular cancer. Liver Int 35: 240-253, 2015

30. Xu J, Li X, Yang H, Chang R, Kong C and Yang L: SIN1 promotes invasion and metastasis of hepatocellular carcinoma by facilitating epithelial-mesenchymal transition. Cancer 119: 2247-2257, 2013.

31. Choi SY, Shin HC, Kim SY and Park YW: Role of TMPRSS4 during cancer progression. Drug News Perspect 21: 417-423, 2008 . 